\title{
Safety of synthetic and biological DMARDs: a systematic literature review informing the 2016 update of the EULAR recommendations for management of rheumatoid arthritis
}

\author{
Sofia Ramiro, ${ }^{1}$ Alexandre Sepriano, ${ }^{1,2}$ Katerina Chatzidionysiou, ${ }^{3}$ Jackie L Nam,,${ }^{4,5}$ \\ Josef S Smolen, ${ }^{6,7}$ Désirée van der Heijde, ${ }^{1}$ Maxime Dougados, ${ }^{8}$ \\ Ronald van Vollenhoven, ${ }^{9}$ Johannes W Bijlsma, ${ }^{10}$ Gerd R Burmester, ${ }^{11}$ \\ Marieke Scholte-Voshaar, ${ }^{12,13}$ Louise Falzon, ${ }^{14}$ Robert B M Landewé ${ }^{9,15}$
}

\begin{abstract}
- Additional material is published online only. To view please visit the journal online (http://dx.doi.org/10.1136/ annrheumdis-2016-210708)

For numbered affiliations see end of article.
\end{abstract}

Correspondence to Dr Sofia Ramiro, Department of Rheumatology, Leiden University Medical Center, Albinusdreef 2, PO Box 9600, Leiden 2300 RC The Netherlands; sofiaramiro@ gmail.com

Received 21 October 2016 Revised 28 December 2016 Accepted 19 February 2017 Published Online First 15 March 2017

\section{Linked}

- http://dx.doi.org/10.1136/ annrheumdis-2016-210715 - http://dx.doi.org/10.1136/ annrheumdis-2016-211005

\section{CrossMark}

\section{To cite: Ramiro $\mathrm{S}$,} Sepriano A,

Chatzidionysiou $\mathrm{K}$, et al. Ann Rheum Dis 2017;76:1093-1101.

\section{ABSTRACT}

Objectives To assess the safety of synthetic ( $\mathrm{s}$ ) and biological (b) disease-modifying antirheumatic drugs (DMARDs) for the management of rheumatoid arthritis (RA) to inform the European League Against Rheumatism recommendations for the management of RA.

Methods Systematic literature review (SLR) of observational studies comparing any DMARD with another intervention for the management of patients with RA. All safety outcomes were included. A comparator group was required for the study to be included. Risk of bias was assessed with the Hayden's tool.

Results Twenty-six observational studies addressing diverse safety outcomes of therapy with bDMARDs met eligibility criteria (15 on serious infections, 4 on malignancies). Substantial heterogeneity precluded metaanalysis. Together with the evidence from the 2013 SLR, based on 15 studies, 7 at low risk of bias, patients on bDMARDs compared with patients on conventional sDMARDs had a higher risk of serious infections (adjusted HR (aHR) 1.1 to 1.8)—without differences across bDMARDs - a higher risk of tuberculosis (aHR 2.7 to 12.5), but no increased risk of infection by herpes zoster. Patients on bDMARDs did not have an increased risk of malignancies in general, lymphoma or nonmelanoma skin cancer, but the risk of melanoma may be slightly increased (aHR 1.5).

Conclusions These findings confirm the known safety pattern of bDMARDs, including both tumour necrosis factor- $\alpha$ inhibitor (TNFi) and non-TNFi, for the treatment of RA.

\section{INTRODUCTION}

The armamentarium nowadays available for the treatment of patients with rheumatoid arthritis (RA) is impressive and has substantially expanded in the last decades. A plethora of conventional synthetic disease-modifying antirheumatic drugs (csDMARDs), biological DMARDs (bDMARDs) and more recently also targeted synthetic DMARDs (tsDMARDs), which can be used in different sequences and/or combinations, is at the disposal of rheumatologists to offer to patients. This, naturally, also implies choices to be made when deciding on the best treatment for a particular patient.

Treatment decisions, particularly in the case of patients with RA with insufficient response to a first csDMARD, are mainly made based on the expected efficacy of a drug. ${ }^{1}$ However, there are no important differences in efficacy across bDMARDs and tsDMARDs. $^{2} 3$ Therefore, other aspects among which safety may have a more prominent place in decision-making. ${ }^{1}$ While short-term safety is addressed in clinical trials, it is long-term safety that we are primarily interested in when making our decisions. Observational studies (eg, cohort studies, registries) provide us with more relevant information since, unlike clinical trials, they include a non-selected group of patients, are representative of daily clinical practice and cover a longer period of time. ${ }^{4}$

In order to inform the task force responsible for the 2016 update of the European League Against Rheumatism (EULAR) RA management recommendations, we performed a systematic literature review (SLR) to update the evidence for the safety of csDMARDs, tsDMARDs and bDMARDs in patients with RA. ${ }^{5}$ This SLR is an update of the SLR performed previously for the corresponding 2013 update of the RA management recommendations. ${ }^{6}$ The results of this and two other SLRs ${ }^{2} 3$ provided the task force with the current state of evidence.

\section{METHODS}

\section{Literature search}

The search was performed in MEDLINE, EMBASE and The Cochrane CENTRAL Register of Controlled Trials (Central), until 9 March 2016, without language restrictions. All newly included studies were published from 2013 onwards, as an update of the previous SLR. ${ }^{6}$ As this SLR is an update of the 2013 SLR, ${ }^{6}$ results are shown together to give a more complete overview on the safety of DMARDs. Details on complete search strategies are provided in online supplementary material. References from included studies were also screened.

The literature search addressed the safety of DMARDs. The research questions were structured 
according to a PICO format (Patients, Intervention, Comparator and Outcomes) and eligible study types were defined. ${ }^{7}$ Participants were adults (aged $\geq 18$ years) with a clinical diagnosis of RA. Studies including patients with other diagnoses were eligible only if the results from patients with RA were presented separately. The intervention was any DMARD (csDMARD, bDMARD—including biosimilars-or tsDMARD), including all drugs (methotrexate, leflunomide, hydroxychloroquine, sulfasalazine, gold/auranofin, azathioprine, chlorambucil, chloroquine, ciclosporin, cyclophosphamide, mycophenolate, minocycline, penicillamine, tacrolimus, anakinra, infliximab, etanercept, adalimumab, rituximab (RTX), abatacept, tocilizumab, golimumab, certolizumab pegol or tofacitinib), formulations and duration). Glucocorticoids were also included. The comparator was a(nother) bDMARD, sDMARD, glucocorticoid, combination therapy or the general population. Studies were only eligible if they included a comparator group, as a formal comparison is the only insightful manner to take any conclusions about safety. All safety outcomes were considered, namely infections (including serious infections, opportunistic infections such as tuberculosis and herpes zoster), malignancies, mortality, cardiovascular disease, change in lipid levels, impairment in renal function, elevation of liver enzymes, haematological abnormalities, gastrointestinal effects, demyelinating disease, induction of autoimmune disease and teratogenicity. Only observational studies were included, namely cohort studies/registries and study series with $>30$ cases.

\section{Selection of studies, data extraction and assessment of risk of bias}

Two reviewers (SR and AS) independently screened titles and abstracts, and if necessary the full-text, for eligibility. In cases of disagreement, a third reviewer (RBML) was involved. Data from eligible studies were extracted regarding study and population characteristics, inclusion and exclusion criteria, follow-up time, interventions, outcome definition and outcome measures using a standardised data extraction form.

The two reviewers independently assessed the risk of bias of each included study using the 'Hayden-tool', which evaluates the following items: participation, attrition, prognostic factor measurement, outcome measurement, confounding and analysis. $^{8}$

\section{RESULTS}

In total and after de-duplication, 4436 references were obtained, of which 26 studies were included (flowchart in online supplementary figure S1). All studies included patients on bDMARDs and only one study also addressed a comparison between csDMARDs. ${ }^{9}$ There were no eligible studies on tsDMARDs or glucocorticoids. Of the included studies, 15 studies focused on infections, ${ }^{10-24} 4$ on malignancies, ${ }^{9}{ }^{25-27} 1$ on mortality, ${ }^{28} 4$ on cardiovascular events ${ }^{29-32}$ and 2 on interstitial lung disease. ${ }^{33}$ Details can be found in tables 1-3 and online supplementary tables S1-S57.

Studies were very heterogeneous in every single item of the PICO, thus precluding data-pooling (meta-analysis), and results are presented descriptively.

Of the newly included 11 studies addressing serious infections, 6 compared patients on bDMARDs with those on csDMARDs or with the general population, ${ }^{10-15}$ whereas 8 studies ${ }^{10-12} \quad{ }^{16-20}$ addressed a comparison between different bDMARDs (3 studies addressed both comparisons) ${ }^{10-12}$ - tables 1 and 2 . In total, and considering the previous evidence from $2013,{ }^{6} 15$ studies, 7 at low risk of bias, compared the risk of serious infections between bDMARDs and csDMARDs and overall found a significantly increased risk with adjusted HRs (aHR) between 1.0 and 1.8 per study. ${ }^{10-15}{ }^{35-43}$ More recent studies at low risk of bias did not show an increased risk. ${ }^{10} 14$ One study comparing bDMARDs with the general population reported standardised incidence rates of 16-20 for tumour necrosis factor- $\alpha$ inhibitor (TNFi) and even higher for RTX (table 1). ${ }^{15}$ In total, six studies, ${ }^{21} 44-48$ performed in European and American datasets, of which four were at low risk of bias, focused on the occurrence of herpes zoster, most of them reporting no increased risk for this type of infection in patients on TNFi (no studies for other bDMARDs), particularly the studies at low risk of bias and/or those that had been adjusted for dropouts. ${ }^{21} 44-46$

Seven studies addressing tuberculosis, most of them being at moderate or high risk of bias, showed an increased risk of tuberculosis in patients on TNFi (no studies for other sDMARDS), both compared with the general population and to patients on csDMARDs (aHR 2.7 to 12.5 per study). ${ }^{1122} 234950$

One study at moderate risk of bias did not show an increased risk of skin infections in patients on TNFi compared with patients on csDMARDs. ${ }^{24}$ One study at moderate risk of bias reported no increased risk of non-viral opportunistic infections in patients on TNFi versus csDMARDs. ${ }^{23}$

Concerning comparisons across bDMARDs, eight studies, only one of them being at low risk of bias, compared the risk of serious infections across bDMARDs and in general did not show differences between several drugs. ${ }^{10-12}$ 16-20 Comparisons included TNFi and non-TNFi, both aggregated in classes and as individual drugs. One of the studies found a signal for a higher risk of serious infections with infliximab compared with etanercept ${ }^{17}$ and another for infliximab, etanercept and RTX compared with abatacept ${ }^{20}$ (table 2). No differences were found between TNFi and non-TNFi on the risk of herpes zoster. $^{21}$

The overall risk of malignancies was investigated in a total of nine studies, six of them being at low risk of bias (table 3). ${ }^{9} 10142551-55$ Both in comparison to the general population and to patients on csDMARDs, patients on bDMARDs did not show an increased risk for malignancies. In a few more recent studies, patients on non-TNFi were also included. ${ }^{9} 10$ Similarly, no increased risk for solid cancers has been found for patients on bDMARDs compared with csDMARDs (two studies were at low risk of bias). ${ }^{96}$ The same was true for the analysis of the individual solid cancers (eg, breast cancer, lung cancer, colorectal cancer -online supplementary table S37.2). Patients on bDMARDs (five studies, three at low risk of bias, all with TNFi), as compared with the general population, had a higher risk of lymphoma, with adjusted aHRs ranging from 2.3 to 5.9 , but in comparison to patients on csDMARDs (three studies, two at low risk of bias), no increased risk was found. In patients on bDMARDs, non-melanoma skin cancer may occur more frequently than in the general population (aHR 1.7; one study at low risk of bias), but compared with csDMARDs, there was no increased risk (four studies, two at low risk of bias). A 'safety alarm signal' was shown for abatacept compared with csDMARDs: a higher risk for its occurrence, with an aHR of 15.3 (95\% CI 2.1 to 114 ), but this 'signal' was only based on two cases. 9 One study at low risk of bias has shown that patients on bDMARDs may have an increased risk for melanoma compared with csDMARDs (aHR 1.5 (95\% CI 1.0 to 2.2$)$ ). ${ }^{56}$

For the remaining outcomes, the scarcity of data precluded definitive conclusions, but new safety signals were absent (see online supplementary tables S39-S57). 


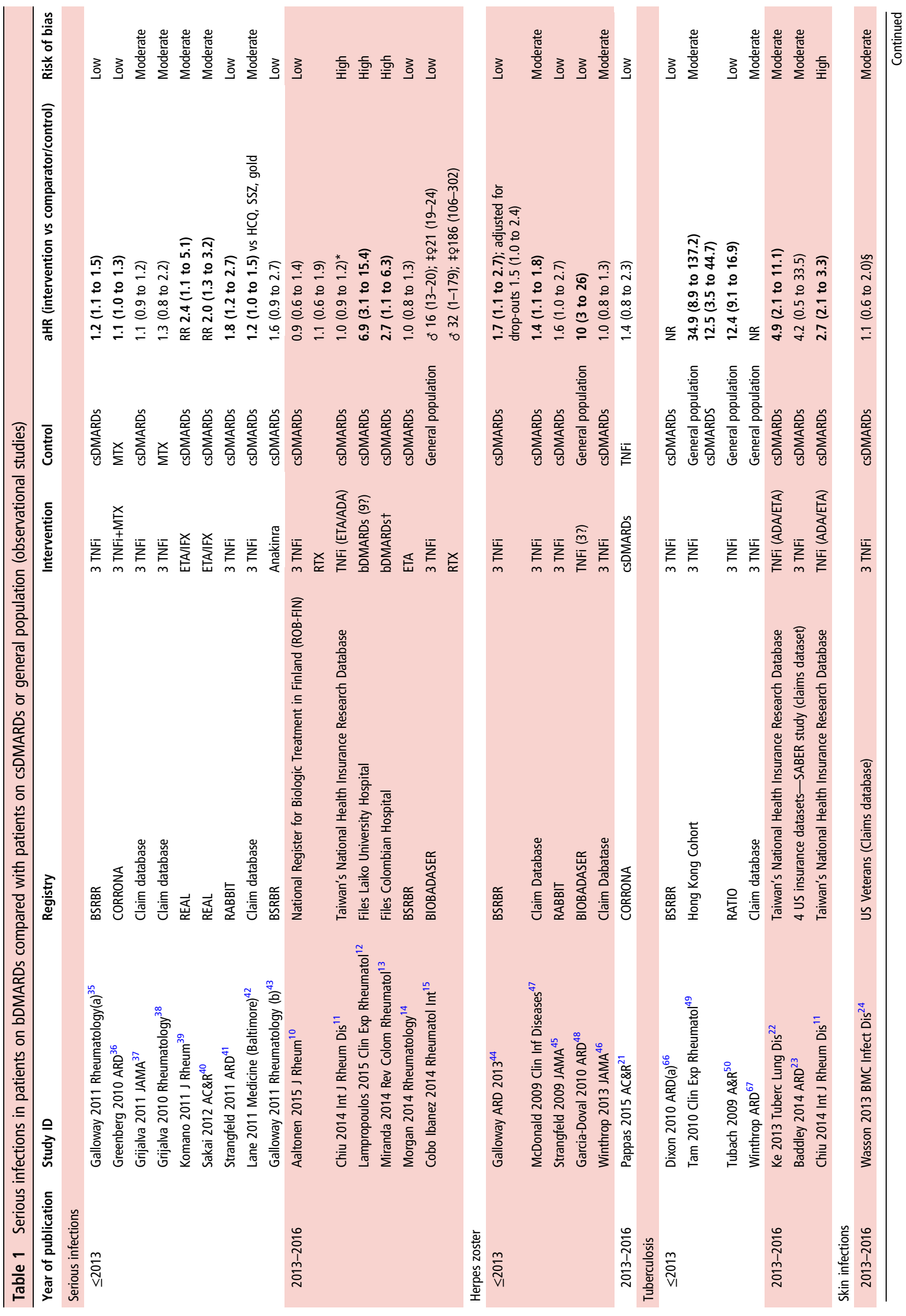


Existing literature has further confirmed that patients on bDMARDs (both TNFi and non-TNFi) have an increased risk of serious infections compared with patients on csDMARDs and that in general there are no differences across bDMARDs. There is an increased risk for tuberculosis with TNFi, whereas this has not been studied well for non-TNFi. There does not seem to be an increased risk of herpes zoster with bDMARDs. In addition, bDMARDs are not associated with an increased risk of malignancies, with the potential exception of melanoma, based on one study only.

Interestingly, more recent studies addressing serious infections, and especially those at low risk of bias, did not show an increased risk of infections anymore. ${ }^{10}{ }^{14}$ This contrasts with earlier studies addressing the same outcome, in which a higher risk of infections had been reported consistently even in those at low risk of bias. ${ }^{35} 3641$ This effect may reflect a change in the attitude of physicians who now more carefully screen and monitor patients (including infection prophylaxis, when indicated) and treat infections in patients on bDMARDs appropriately.

In general, our conclusions are in line with those drawn in $2013,{ }^{6}$ which is reassuring. The accumulating body of evidence related to bDMARDs is consistently showing us that patients with RA can be treated in a relatively safe way with these drugs. This SLR extends these conclusions also to non-TNFi bDMARDs, which was not possible in the previous SLR. ${ }^{6}$ Still, most literature on safety pertains to TNFi, and we need more studies including non-TNFi bDMARDs and tsDMARDs in future.

This SLR also highlights the importance of observational studies in addressing safety aspects of treatment, particularly those studies that include a comparator and truly allow us to assign risks to patients on a particular intervention (eg, bDMARDs). Without a proper comparison, it is impossible to truly judge risks. In addition, these are studies that include all types of patients and follow them up for a long period of time, directly reflecting daily clinical practice, which increases their generalisability. ${ }^{4}$ This is what we need to get better insight into safety aspects of treatments as it complements the limited information derived from clinical trials. Admittedly, conducting this type of analysis in observational studies properly is challenging. ${ }^{57}$ Several confounders can influence the relationships of interest, and they need to be carefully considered. Even though this is done, even the 'best comparator' that we at the moment have to contrast safety of bDMARDs with, namely csDMARDs, also implies challenges and limitations, as we know that patients on csDMARDs have less severe disease, or sometimes historical data are used for comparison purposes, which also introduces some sources of bias. Increasingly complex analyses are being undertaken to circumvent the known challenges, for example, analysis adjusted for propensity score. ${ }^{58}$ Collaborations between registries are important in order to homogenise procedures, raise the overall quality and allow comparisons, and these should be encouraged. ${ }^{59}$ This will lead to better information for clinicians and better care to patients. Over and above the current data from observational studies, other information previously obtained through randomised clinical trials (RCTs) or addressed in package inserts should be taken into account. The labels of each drug, including adverse events and lab monitoring, remain undisputed and it is good practice to follow them.

Although this SLR aimed at including all DMARDs, the eligible studies were only on bDMARDs. This points to the need 


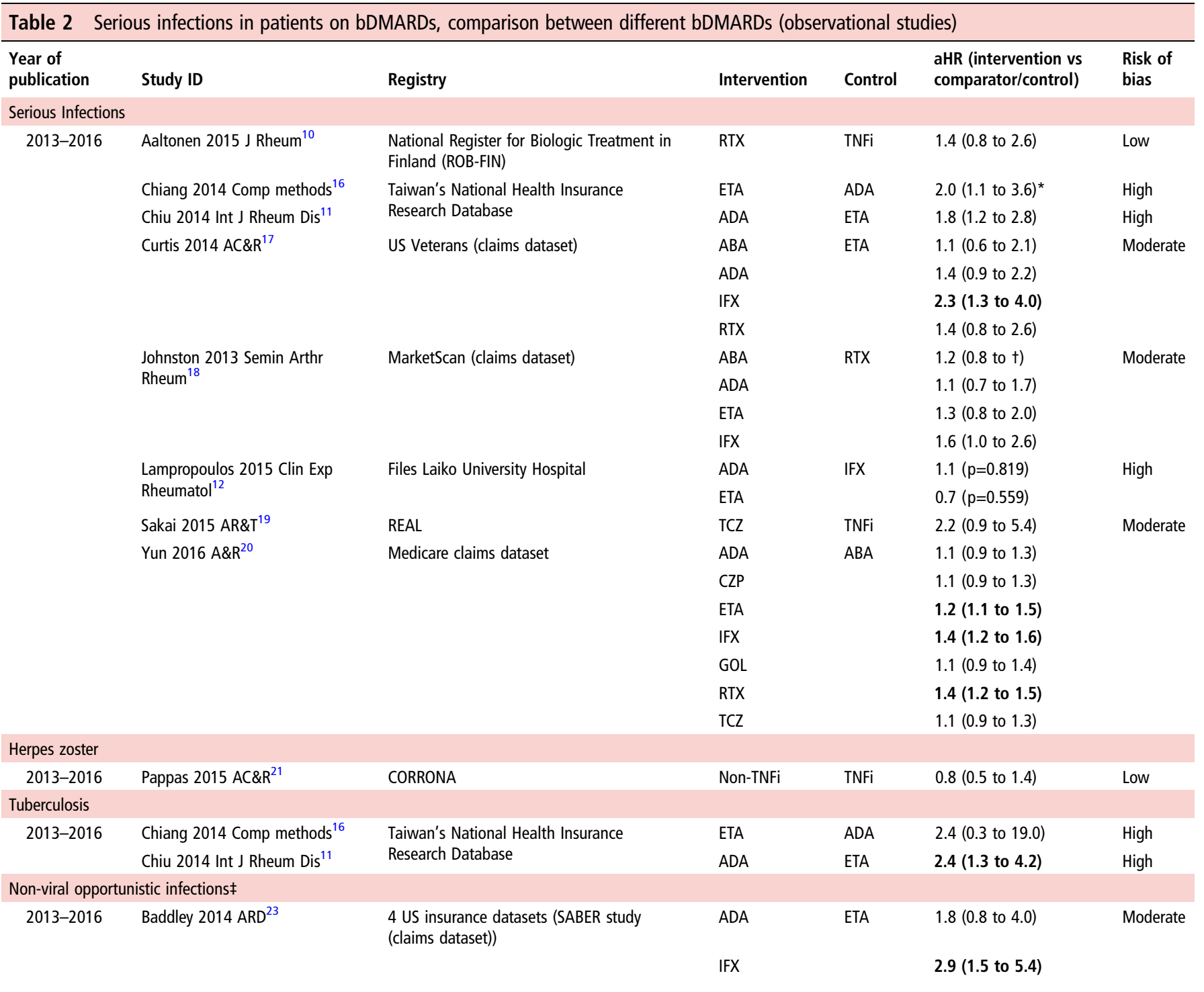

Estimates in bold reflect a risk/ratio statistically significantly different from 1, ie association is statistically significant.

More details are found in online supplementary tables S1-S31.

*Unadjusted estimate; no adjusted estimate reported.

tNo upper border of $\mathrm{Cl}$ given.

‡Non-viral opportunistic infections included fungal infections, tuberculosis, pneumocystosis, nocardiosis/actinomycosis, non-tuberculous mycobacteria, salmonellosis, listeriosis and legionellosis.

ABA, abatacept; ADA, adalimumab; aHR, adjusted adjusted Hazard Ratio; CORRONA, Consortium of Rheumatology Researchers of North America; CZP, certolizumab pegol; ETA, etanercept; GOL, golimumab; IFX, infliximab; REAL, Registry of Japanese Rheumatoid Arthritis Patients for Long-term Safety; RTX, rituximab; TCZ, tocilizumab; TNFi, tumour necrosis factor $\alpha$ inhibitor.

for good quality safety studies addressing the remaining DMARDs. Only one study included a comparison between csDMARDs and the focus of that study was still on bDMARDs. ${ }^{9}$ Among the studies on bDMARDs, none of them included patients on biosimilars (yet). In addition, observational studies addressing tsDMARDs (Jak inhibitor(s)) have not yet been found. However, RCT data point towards a higher risk of serious infections, infections caused by herpes zoster and tuberculosis, risks that should not be ignored and that warrant further research. ${ }^{2} 60$ Finally, while glucocorticoids are gaining importance as bridging treatment for RA, no single study meeting the eligibility criteria could be found. Nevertheless, concerns regarding the long-term safety of glucocorticoids remain, ${ }^{2}$ and recent studies, even though some of them are uncontrolled or may suffer from confounding by indication, point towards a higher cardiovascular risk, a higher risk of infections and higher mortality in patients taking glucocorticoids. $^{28}$ 61-64 These are all questions that should be addressed, likely in registries, and with the use of analytical techniques that have previously been used with success in safety studies with bDMARDs.

In line with the frequent updates of the EULAR recommendations for the management of RA, it is to expect that an update of this SLR will soon deserve careful attention, particularly if the above-mentioned unmet needs are fulfilled and more good quality safety registry data, and covering more interventions, become available. An example is the recent study from Strangfeld et $a l^{65}$ showing a higher risk of lower intestinal perforation in patients taking tocilizumab compared with patients on csDMARDs, which has no longer been included in this SLR because it was accepted for publication after the update of the search for this SLR and when the task force meeting for the EULAR recommendations on the management of RA had already taken place. This and other 


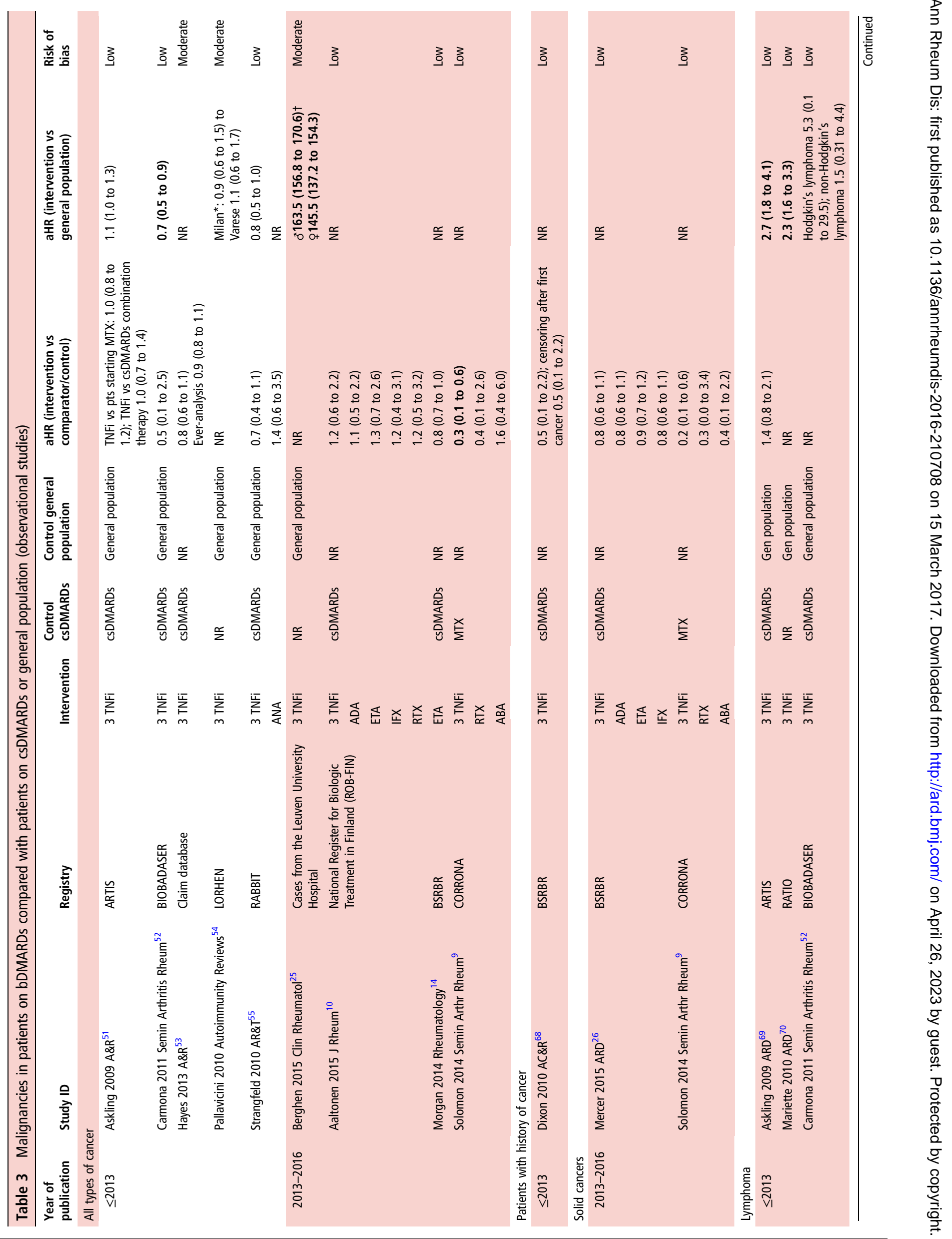




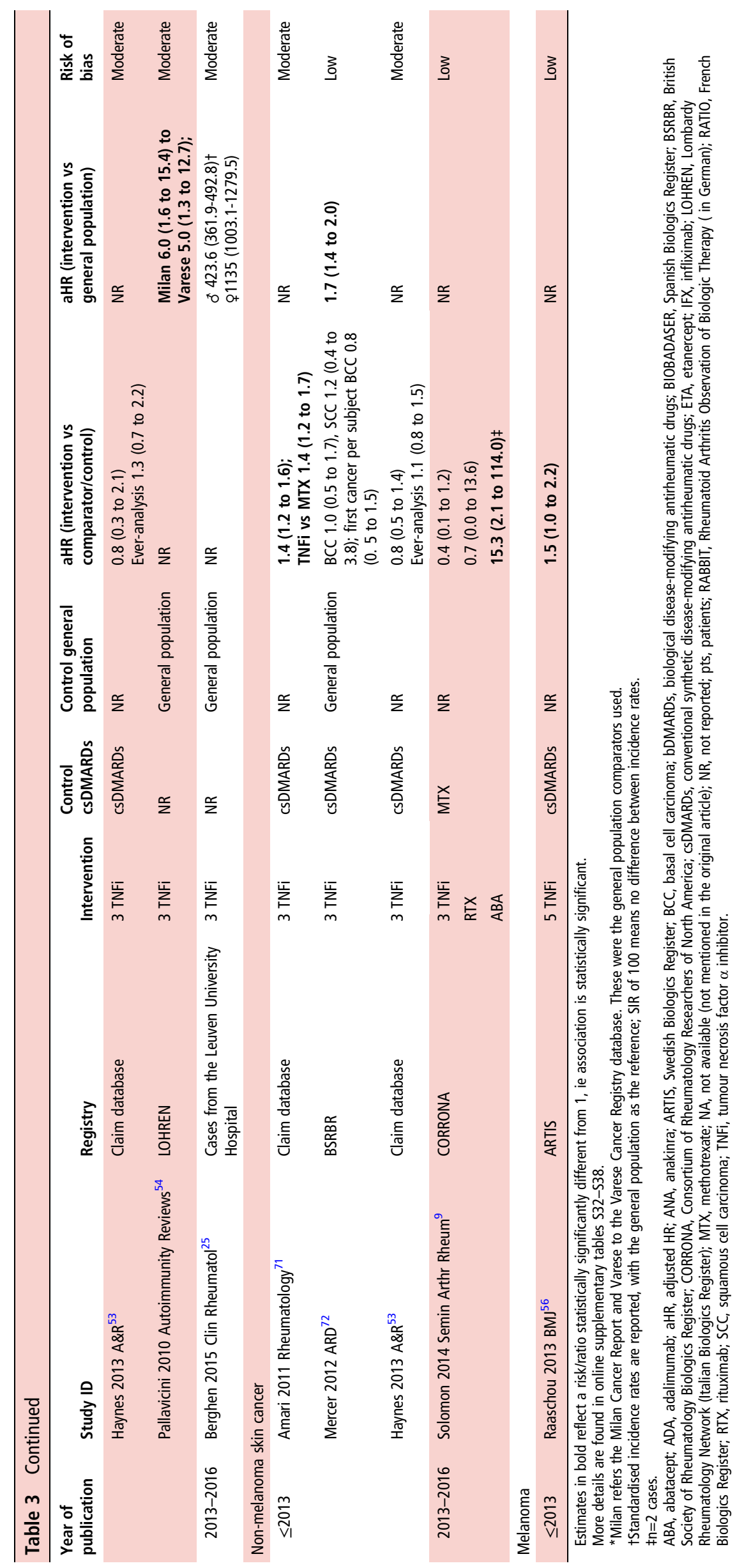


relevant studies should be considered in a future update of this SLR.

\section{Author affiliations}

${ }^{1}$ Department of Rheumatology, Leiden University Medical Center, Leiden, The Netherlands

${ }^{2}$ CEDOC, Nova Medical School, Universidade Nova de Lisboa, Lisboa, Portugal ${ }^{3}$ Unit for Clinical Therapy Research, Inflammatory Diseases (ClinTRID), The Karolinska Institute, Stockholm, Sweden

${ }^{4}$ Leeds Institute of Rheumatic and Musculoskeletal Medicine, University of Leeds, Chapel Allerton Hospital Leeds, Leeds, UK

${ }^{5}$ NIHR Leeds Musculoskeletal Biomedical Research Unit, Leeds Teaching Hospitals NHS Trust, Leeds, UK

${ }^{6}$ Division of Rheumatology, Department of Medicine 3, Medical University of Vienna, Vienna, Austria

${ }^{7}$ Department of Medicine, Hietzing Hospital, Vienna, Austria

${ }^{8}$ Department of Rheumatology, Paris Descartes University, Cochin Hospital, Assistance Publique-Hôpitaux de Paris, INSERM (U1153): Clinical Epidemiology and Biostatistics, Paris, France

${ }^{9}$ Department of Clinical Immunology \& Rheumatology, Amsterdam Rheumatology Center, Amsterdam, The Netherlands

${ }^{10}$ Department of Rheumatology, University Medical Center Utrecht, Utrecht, The Netherlands

${ }^{11}$ Department of Rheumatology and Clinical Immunology, Charité-University Medicine Berlin, Berlin, Germany

${ }^{12}$ Department of Psychology, Health and Technology, University of Twente, Enschede, The Netherlands

${ }^{13}$ EULAR Standing Committee of People with Arthritis/Rheumatism in Europe

${ }^{14}$ Center for Behavioral Cardiovascular Health, Columbia University Medical Center, New York, USA

${ }^{15}$ Department of Rheumatology, Zuyderland Medical Center, Heerlen,

The Netherlands

Contributors All authors contributed and finally approved the current manuscript.

Competing interests JS, Amgen, AbbVie, AstraZeneca, Astro, BMS, Celgene, GlaxoSmithKline, ILTOO, Janssen, Merck-Serono, MSD, Novartis-Sandoz, Pfizer, Roche-Chugai, Samsung, UCB. DvdH, AbbVie, Amgen, Astellas, AstraZeneca, BMS, Celgene, Daiichi, Eli-Lilly, Galapagos, Gilead, Merck, Novartis, Pfizer, Roche, Sanofi-Aventis, UCB, 5, Director of Imaging Rheumatology BV. MD, AbbVie, Pfizer, Novartis, MSD. RvV, AbbVie, Bristol-Myers Squibb, GlaxoSmithKline, Pfizer, Roche, UCB Pharma, Biotest, Janssen, Eli-Lilly, Merck, Vertex. JWB, Roche, AbbVie, Bristol-Myers Squibb, Merck Sharp \& Dohme, Pfizer and UCB. GB, UCB, 2, AbbVie, BMS, Hexal, Janssen, Eli-Lilly, MSD, Medimmune, Novartis, Pfizer, Sanofi-Aventis, Roche. RL, AbbVie, Amgen, Centocor, Novartis, Pfizer, Roche, Schering-Plough, UCB, Pfizer, Ablynx, Amgen, AstraZeneca, Bristol-Myers Squibb, Celgene, Janssen (formerly Centocor), Galapagos, GlaxoSmithKline, Novartis, Novo-Nordisk, Merck, TiGenix, Rheumatology Consultancy BV.

Provenance and peer review Not commissioned; externally peer reviewed.

\section{REFERENCES}

1 Hifinger $\mathrm{M}$, Hiligsmann $\mathrm{M}$, Ramiro $\mathrm{S}$, et al. Economic considerations and patients preferences affect treatment selection for patients with rheumatoid arthritis: a discrete choice experiment among European rheumatologists. Ann Rheum Dis 2017:76:126-32

2 Chatzidionysiou K, Sharzad E, Nam J, et al. Efficacy and safety of glucocorticoids, conventional and targeted synthetic disease-modifying antirheumatic drugs: a systematic literature review and meta-analysis informing the 2016 update of the EULAR recommendations for the management of rheumatoid arthritis. Ann Rheum Dis 2017. doi: 10.1136/annrheumdis-2016-210711.

3 Nam J, Takase-Minegishi K, Ramiro S, et al. Efficacy of biological DMARDs-a systematic literature review informing the 2016 update of the EULAR recommendations for management of rheumatoid arthritis. Ann Rheum Dis 2017 doi: 10.1136/annrheumdis-2016-210711.

4 Zink A, Askling J, Dixon WG, et al. European biologicals registers: methodology, selected results and perspectives. Ann Rheum Dis 2009:68:1240-6.

5 Smolen JS, Landewé R, Breedveld FC, et al. EULAR recommendations for the management of rheumatoid arthritis with synthetic and biological disease modifying antirheumatic drugs - 2016 update. Ann Rheum Dis 2017. doi: 10.1136/ annrheumdis-2016-210715. [Epub ahead of print 6 Mar 2017].

6 Ramiro S, Gaujoux-Viala C, Nam JL, et al. Safety of synthetic and biological DMARDs: a systematic literature review informing the 2013 update of the EULAR recommendations for management of rheumatoid arthritis. Ann Rheum Dis 2014;73:529-35

7 Sackett D, WS R, Rosenberg W, et al. Evidence-based medicine: how to practice and teach EBM. London: Churchill Livingstone, 1997.
8 Hayden JA, Cote P, Bombardier C. Evaluation of the quality of prognosis studies in systematic reviews. Ann Intern Med 2006:144:427-37.

9 Solomon DH, Kremer JM, Fisher M, et al. Comparative cancer risk associated with methotrexate, other non-biologic and biologic disease-modifying anti-rheumatic drugs. Semin Arthritis Rheum 2014;43:489-97.

10 Aaltonen KJ, Joensuu JT, Virkki L, et al. Rates of serious infections and malignancies among patients with rheumatoid arthritis receiving either tumor necrosis factor inhibitor or rituximab therapy. J Rheumatol 2015:42:372-8.

11 Chiu YM, Lang HC, Lin HY, et al. Risk of tuberculosis, serious infection and lymphoma with disease-modifying biologic drugs in rheumatoid arthritis patients in Taiwan. Int J Rheum Dis 2014;17(Suppl 3):9-19.

12 Lampropoulos CE, Orfanos P, Bournia VK, et al. Adverse events and infections in patients with rheumatoid arthritis treated with conventional drugs or biologic agents: a real world study. Clin Exp Rheumatol 2015;33:216-24

13 Miranda JV, Peñaranda LFP, Grajales CM, et al. Infections in rheumatoid arthritis patients: biological therapy versus disease modifying anti-rheumatic drugs: one year follow-up. Revista Colombiana de Reumatologia 2014:21:27-34.

14 Morgan CL, Emery P, Porter D, et al. Treatment of rheumatoid arthritis with etanercept with reference to disease-modifying anti-rheumatic drugs: long-term safety and survival using prospective, observational data. Rheumatology 2014;53:186-94.

15 Cobo-lbáñez T, Descalzo MA, Loza-Santamaría E, et al. Serious infections in patients with rheumatoid arthritis and other immune-mediated connective tissue diseases exposed to anti-TNF or rituximab: data from the Spanish registry BIOBADASER 2.0. Rheumatol Int 2014;34:953-61.

16 Chiang YC, Kuo LN, Yen YH, et al. Infection risk in patients with rheumatoid arthritis treated with etanercept or adalimumab. Comput Methods Programs Biomed 2014:116:319-27.

17 Curtis JR, Yang S, Patkar NM, et al. Risk of hospitalized bacterial infections associated with biologic treatment among US veterans with rheumatoid arthritis. Arthritis Care and Research 2014;66:990-7.

18 Johnston SS, Turpcu A, Shi N, et al. Risk of infections in rheumatoid arthritis patients switching from anti-TNF agents to rituximab, abatacept, or another anti-TNF agent, a retrospective administrative claims analysis. Semin Arthritis Rheum 2013;43:39-47.

19 Sakai R, Cho SK, Nanki T, et al. Head-to-head comparison of the safety of tocilizumab and tumor necrosis factor inhibitors in rheumatoid arthritis patients (RA) in clinical practice: results from the registry of Japanese RA patients on biologics for long-term safety (REAL) registry. Arthritis Res Ther 2015:17:74.

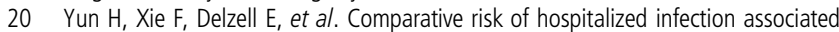
with biologic agents in rheumatoid arthritis patients enrolled in Medicare. Arthritis Rheumatol 2016:68:56-66.

21 Pappas DA, Hooper MM, Kremer JM, et al. Herpes zoster reactivation in patients with rheumatoid arthritis: analysis of disease characteristics and disease-modifying antirheumatic drugs. Arthritis Care Res (Hoboken) 2015;67:1671-8.

22 Ke WM, Chen LS, Parng IM, et al. Risk of tuberculosis in rheumatoid arthritis patients on tumour necrosis factor-alpha inhibitor treatment in Taiwan. Int J Tuberc Lung Dis 2013:17:1590-5.

23 Baddley JW, Winthrop KL, Chen L, et al. Non-viral opportunistic infections in new users of tumour necrosis factor inhibitor therapy: results of the SAfety Assessment of Biologic ThERapy (SABER) study. Ann Rheum Dis 2014;73:1942-8.

24 Wasson NJ, Varley CD, Schwab P, et al. "Serious skin \& soft tissue infections in rheumatoid arthritis patients taking anti-tumor necrosis factor alpha drugs: a nested case-control study". BMC Infect Dis 2013;13:533.

25 Berghen N, Teuwen LA, Westhovens $\mathrm{R}$, et al. Malignancies and anti-TNF therapy in rheumatoid arthritis: a single-center observational cohort study. Clin Rheumatol 2015:34:1687-95.

26 Mercer LK, Lunt M, Low AL, et al. Risk of solid cancer in patients exposed to anti-tumour necrosis factor therapy: results from the British Society for Rheumatology Biologics Register for Rheumatoid Arthritis. Ann Rheum Dis 2015;74:1087-93.

27 Phillips C, Zeringue AL, McDonald JR, et al. Tumor necrosis factor inhibition and head and neck cancer recurrence and death in rheumatoid arthritis. PLOS ONE 2015:10:e0143286.

28 Listing J, Kekow J, Manger B, et al. Mortality in rheumatoid arthritis: the impact of disease activity, treatment with glucocorticoids, TNF $\alpha$ inhibitors and rituximab. Ann Rheum Dis 2015;74:415-21.

29 Ogdie A, Yu Y, Haynes K, et al. Risk of major cardiovascular events in patients with psoriatic arthritis, psoriasis and rheumatoid arthritis: a population-based cohort study. Ann Rheum Dis 2015:74:326-32.

30 Solomon $\mathrm{DH}$, Rassen JA, Kuriya $\mathrm{B}$, et al. Heart failure risk among patients with rheumatoid arthritis starting a TNF antagonist. Ann Rheum Dis 2013;72:1813-18.

31 Desai RJ, Eddings W, Liao KP, et al. Disease-modifying antirheumatic drug use and the risk of incident hyperlipidemia in patients with early rheumatoid arthritis: a retrospective cohort study. Arthritis Care Res (Hoboken) 2015;67:457-66.

32 Kim SC, Solomon DH, Liu J, et al. Risk of Venous thromboembolism in patients with rheumatoid arthritis: initiating disease-modifying antirheumatic drugs. Am J Med 2015; 128:539.e7-39.e17 
33 Herrinton LJ, Harrold LR, Liu L, et al. Association between anti-TNF- $\alpha$ therapy and interstitial lung disease. Pharmacoepidemiol Drug Saf 2013;22:394-402.

34 Curtis JR, Sarsour K, Napalkov P, et al. Incidence and complications of interstitial lung disease in users of tocilizumab, rituximab, abatacept and anti-tumor necrosis factor $\alpha$ agents, a retrospective cohort study. Arthritis Res Ther 2015;17:319.

35 Galloway JB, Hyrich KL, Mercer LK, et al. Anti-TNF therapy is associated with an increased risk of serious infections in patients with rheumatoid arthritis especially in the first 6 months of treatment: updated results from the British Society for Rheumatology Biologics Register with special emphasis on risks in the elderly. Rheumatology (Oxf) 2011;50:124-31.

36 Greenberg JD, Reed G, Kremer JM, et al. Association of methotrexate and tumour necrosis factor antagonists with risk of infectious outcomes including opportunistic infections in the CORRONA registry. Ann Rheum Dis 2010;69:380-6.

37 Grijalva CG, Chen L, Delzell $E$, et al. Initiation of tumor necrosis factor- $\alpha$ antagonists and the risk of hospitalization for infection in patients with autoimmune diseases. JAMA 2011;306:2331-9.

38 Grijalva CG, Kaltenbach L, Arbogast PG, et al. Initiation of rheumatoid arthritis treatments and the risk of serious infections. Rheumatology (Oxf) 2010;49: 82-90.

39 Komano $Y$, Tanaka M, Nanki T, et al. Incidence and risk factors for serious infection in patients with rheumatoid arthritis treated with tumor necrosis factor inhibitors: a report from the Registry of Japanese Rheumatoid Arthritis Patients for Long-term Safety. J Rheumatol 2011;38:1258-64.

40 Sakai R, Komano Y, Tanaka M, et al. Time-dependent increased risk for serious infection from continuous use of tumor necrosis factor antagonists over three years in patients with rheumatoid arthritis. Arthritis Care Res (Hoboken) 2012;64:1125-34.

41 Strangfeld A, Eveslage M, Schneider M, et al. Treatment benefit or survival of the fittest: what drives the time-dependent decrease in serious infection rates under TNF inhibition and what does this imply for the individual patient? Ann Rheum Dis 2011;70:1914-20.

42 Lane MA, McDonald JR, Zeringue AL, et al. TNF- $\alpha$ antagonist use and risk of hospitalization for infection in a national cohort of veterans with rheumatoid arthritis. Medicine (Baltim) 2011;90:139-45

43 Galloway JB, Hyrich $\mathrm{KL}$, Mercer LK, et al. The risk of serious infections in patients receiving anakinra for rheumatoid arthritis: results from the British Society for Rheumatology Biologics Register. Rheumatology (Oxf) 2011;50:1341-2.

44 Galloway JB, Mercer LK, Moseley A, et al. Risk of skin and soft tissue infections (including shingles) in patients exposed to anti-tumour necrosis factor therapy: results from the British Society for Rheumatology Biologics Register. Ann Rheum Dis 2013;72:229-34

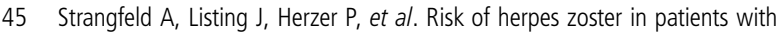
rheumatoid arthritis treated with anti-TNF-alpha agents. 2009;301:737-44.

46 Winthrop KL, Baddley JW, Chen L, et al. Association between the initiation of anti-tumor necrosis factor therapy and the risk of herpes zoster. JAMA 2013:309:887-95.

47 McDonald JR, Zeringue AL, Caplan L, et al. Herpes zoster risk factors in a national cohort of veterans with rheumatoid arthritis. Clin Infect Dis 2009:48:1364-71.

48 García-Doval I, Pérez-Zafrilla B, Descalzo MA, et al. Incidence and risk of hospitalisation due to shingles and chickenpox in patients with rheumatic diseases treated with TNF antagonists. Ann Rheum Dis 2010;69:1751-5.

49 Tam LS, Leung CC, Ying SK, et al. Risk of tuberculosis in patients with rheumatoid arthritis in Hong Kong-the role of TNF blockers in an area of high tuberculosis burden. Clin Exp Rheumatol 2010;28:679-85

50 Tubach F, Salmon D, Ravaud P, et al. Risk of tuberculosis is higher with anti-tumor necrosis factor monoclonal antibody therapy than with soluble tumor necrosis facto receptor therapy: the three-year prospective French Research Axed on Tolerance of Biotherapies registry. Arthritis Rheum 2009;60:1884-94.

51 Askling J, van Vollenhoven RF, Granath F, et al. Cancer risk in patients with rheumatoid arthritis treated with anti-tumor necrosis factor alpha therapies: does the risk change with the time since start of treatment? Arthritis Rheum 2009;60:3180-9.

52 Carmona L, Abasolo L, Descalzo MA, et al. Cancer in patients with rheumatic diseases exposed to TNF antagonists. Semin Arthritis Rheum 2011;41:71-80.
53 Haynes K, Beukelman T, Curtis JR, et al. Tumor necrosis factor $\alpha$ inhibitor therapy and cancer risk in chronic immune-mediated diseases. Arthritis Rheum 2013;65:48-58

54 Pallavicini FB, Caporali R, Sarzi-Puttini $P$, et al. Tumour necrosis factor antagonist therapy and cancer development: analysis of the LORHEN registry. Autoimmun Rev 2010;9:175-80.

55 Strangfeld A, Hierse F, Rau R, et al. Risk of incident or recurrent malignancies among patients with rheumatoid arthritis exposed to biologic therapy in the German biologics register RABBIT. Arthritis Res Ther 2010;12:R5.

56 Raaschou P, Simard JF, Holmqvist M, et al. Rheumatoid arthritis, anti-tumour necrosis factor therapy, and risk of malignant melanoma: nationwide population based prospective cohort study from Sweden. BMJ 2013;346:f1939.

57 Dixon WG, Carmona L, Finckh A, et al. EULAR points to consider when establishing, analysing and reporting safety data of biologics registers in rheumatology. Ann Rheum Dis 2010;69:1596-602.

58 Austin PC. An introduction to propensity score methods for reducing the effects of confounding in observational studies. Multivariate Behav Res 2011;46:399-424.

59 Kearsley-Fleet L, Závada J, Hetland ML, et al. The EULAR study group for registers and observational drug studies: comparability of the patient case mix in the European biologic disease modifying anti-rheumatic drug registers. Rheumatology (Oxf) 2015;54:1074-9.

60 Winthrop KL, Park SH, Gul A, et al. Tuberculosis and other opportunistic infections in tofacitinib-treated patients with rheumatoid arthritis. Ann Rheum Dis 2016:75:1133-8

61 de Hair $\mathrm{M}$, IJff $\mathrm{N}$, Jacobs J, et al. Long-term adverse events after daily concomitant treatment with $10 \mathrm{mg}$ prednisone in the 2-year computer assisted management in early rheumatoid arthritis trial-II. Arthritis Rheumatol 2015;67.

62 Richter $\mathrm{A}$, Listing J, Schneider $\mathrm{M}$, et al. Impact of treatment with biologic DMARDs on the risk of sepsis or mortality after serious infection in patients with rheumatoid arthritis. Ann Rheum Dis 2016;75:1667-73.

63 Roubille $C$, Richer V, Starnino T, et al. The effects of tumour necrosis factor inhibitors, methotrexate, non-steroidal anti-inflammatory drugs and corticosteroids on cardiovascular events in rheumatoid arthritis, psoriasis and psoriatic arthritis: a systematic review and meta-analysis. Ann Rheum Dis 2015;74:480-9.

64 del Rincón I, Battafarano DF, Restrepo JF, et al. Glucocorticoid dose thresholds associated with all-cause and cardiovascular mortality in rheumatoid arthritis. Ann Rheum Dis 2014;66:264-72.

65 Strangfeld A, Richter A, Siegmund B, et al. Risk for lower intestinal perforations in patients with rheumatoid arthritis treated with tocilizumab in comparison to treatment with other biologic or conventional synthetic DMARDs. Ann Rheum Dis 2017;76:504-10

66 Dixon WG, Hyrich KL, Watson KD, et al. Drug-specific risk of tuberculosis in patients with rheumatoid arthritis treated with anti-TNF therapy: results from the British Society for Rheumatology Biologics Register (BSRBR). Ann Rheum Dis 2010;69:522-8

67 Winthrop KL, Baxter R, Liu L, et al. Mycobacterial diseases and antitumour necrosis factor therapy in USA. Ann Rheum Dis 2013;72:37-42.

68 Dixon WG, Watson KD, Lunt $M$, et al. Influence of anti-tumor necrosis factor therapy on cancer incidence in patients with rheumatoid arthritis who have had a prior malignancy: results from the British Society for Rheumatology Biologics Register. Arthritis Care Res (Hoboken) 2010;62:755-63.

69 Askling J, Baecklund E, Granath F, et al. Anti-tumour necrosis factor therapy in rheumatoid arthritis and risk of malignant lymphomas: relative risks and time trends in the Swedish Biologics Register. Ann Rheum Dis 2009;68:648-53.

70 Mariette $\mathrm{X}$, Tubach $\mathrm{F}$, Bagheri $\mathrm{H}$, et al. Lymphoma in patients treated with anti-TNF: results of the 3-year prospective French RATIO registry. Ann Rheum Dis 2010;69:400-8

71 Amari W, Zeringue AL, McDonald JR, et al. Risk of non-melanoma skin cancer in a national cohort of veterans with rheumatoid arthritis. Rheumatology (Oxf) 2011;50:1431-9

72 Mercer LK, Green AC, Galloway JB, et al. The influence of anti-TNF therapy upon incidence of keratinocyte skin cancer in patients with rheumatoid arthritis: longitudinal results from the British Society for Rheumatology Biologics Register. Ann Rheum Dis 2012;71:869-74. 